\title{
Phase II Study of Lenvatinib in Patients With Progressive, Recurrent or Metastatic Adenoid Cystic Carcinoma
}

Vatche Tchekmedyian, MD, MEd ${ }^{1}$; Eric J. Sherman, MD ${ }^{1,2}$; Lara Dunn, MD ${ }^{1,2}$; Crystal Tran ${ }^{1}$; Shrujal Baxi, MD, MPH ${ }^{3}$; Nora Katabi, MD ${ }^{1}$; Cristina R. Antonescu, MD ${ }^{1}$; Irina Ostrovnaya, $\mathrm{PhD}^{1}$; Sofia S. Haque, MD ${ }^{1,2}$; David G. Pfister, MD ${ }^{1,2}$; and Alan L. Ho, MD, PhD ${ }^{1,2}$

PURPOSE Recurrent or metastatic adenoid cystic carcinoma (R/M ACC) is a malignant neoplasm of predominantly salivary gland origin for which effective therapies are lacking. We conducted a phase II trial evaluating the multitargeted tyrosine kinase inhibitor lenvatinib in patients with R/M ACC.

PATIENTS AND METHODS This study was conducted with a two-stage minimax design. Patients with histologically confirmed R/M ACC of any primary site with radiographic and/or symptomatic progression were eligible. Any prior therapy was allowed except previous lenvatinib. Patients received lenvatinib 24 mg orally per day. The primary end point was overall response rate. Secondary end points were progression-free survival and safety. An exploratory analysis of how MYB expression and genomic alterations relate to outcomes was conducted.

RESULTS Thirty-three patients were enrolled; 32 were evaluable for the primary end point. Five patients (15.6\%) had a confirmed partial response, 24 patients (75\%) had stable disease, two patients (6.3\%) discontinued treatment as a result of toxicity before the first scan, and one patient (3.1\%) had progression of disease as best response. Median progression-free survival time was 17.5 months (95\% Cl, 7.2 months to not reached), although only eight progression events were observed. Patients otherwise were removed for toxicity $(n=5)$, as a result of withdrawal of consent $(n=9)$, or at the treating physician's discretion $(n=6)$. Twenty-three patients required at least one dose modification, and 18 of 32 patients discontinued lenvatinib for drug-related issues. The most common grade 3 or 4 adverse events were hypertension ( $n=9 ; 28.1 \%$ ) and oral pain $(n=3 ; 9.4 \%$ ). Three grade 4 adverse events were observed (myocardial infarction, $n=1$; posterior reversible encephalopathy syndrome, $\mathrm{n}=1$; and intracranial hemorrhage, $\mathrm{n}=1$ ).

CONCLUSION This trial met the prespecified overall response rate primary end point, demonstrating antitumor activity with lenvatinib in R/M ACC patients. Toxicity was comparable to previous studies, requiring monitoring and management.

\section{J Clin Oncol 37:1529-1537. ๑ 2019 by American Society of Clinical Oncology}

ASSOCIATED

CONTENT

Appendix

Data Supplement

Author affiliations

and support

information (if

applicable) appear at the end of this article.

Accepted on February 25, 2019 and published at jco.org on April 2, 2019: DOI https://doi.org/10. 1200/JC0.18.01859

\section{INTRODUCTION}

Adenoid cystic carcinomas (ACCs) are malignant neoplasms that arise most commonly from major and minor salivary glands and less commonly from other sites. Recurrent or metastatic (R/M) ACC can have a variable course, ${ }^{1}$ and effective treatments are lacking. Prior studies of multitargeted tyrosine kinase inhibitors (TKIs) have yielded modest signs of efficacy with response rates ranging from $0 \%$ to $11 \%$, as listed in Appendix Table A1 (online only). ${ }^{2-9}$

Approximately $50 \%$ of ACCs, regardless of primary site, are characterized by a $t(6 ; 9)(q 22-23 ; p 23-24)$ translocation that conjoins the two transcription factors NFIB and MYB. ${ }^{10-12}$ Even in the absence of the
MYB-NFIB translocation, MYB is overexpressed in a majority of ACCs, suggesting translocation-independent mechanisms of MYB overexpression. ${ }^{13}$ The functional significance of $M Y B$ overexpression is an upregulation of several target genes, including putatively vascular endothelial growth factor A (VEGFA), fibroblast growth factor 2 (FGF2), and KIT beyond what is seen in normal salivary gland tissue. ${ }^{10}$

Lenvatinib is an oral, multitargeted TKI approved for the treatment of progressive, radioiodine-refractory thyroid cancer and unresectable hepatocellular carcinoma with significant inhibitory activity against vascular endothelial growth factor receptors (VEGFRs) 1 to 3 , fibroblast growth factor receptors (FGFRs) 1 to 
3, KIT, platelet-derived growth factor receptors $\alpha$ and $\beta$, and RET. ${ }^{14-18}$ Given the proposed relevance of several of these genes to ACC biology, we conducted a phase II trial evaluating the efficacy of lenvatinib in progressive R/M ACC.

\section{PATIENTS AND METHODS}

\section{Study Design and Patients}

This was an open-label, single-arm, phase II trial conducted at Memorial Sloan Kettering Cancer Center (MSKCC; New York, NY). Patients with pathologically or cytologically confirmed, incurable R/M ACC were eligible if they met the following criteria: age 18 years or older; measurable disease as defined by Response Evaluation Criteria in Solid Tumor (RECIST) version $1.1^{19}$; new or progressive lesion(s) on a radiologic study and/or new or worsening disease-related symptoms detected within 6 months before study enrollment; Eastern Cooperative Oncology Group performance status of 0 or 1 ; and adequate organ and bone marrow function. Any prior therapies for R/M ACC, except lenvatinib, were allowed. Exclusion criteria included symptomatic, metastatic brain or leptomeningeal tumors (asymptomatic or treated tumors were allowed); thrombotic or embolic venous or arterial events within 6 months of study treatment; or proteinuria of greater than $1 \mathrm{~g}$ per 24 hours. Complete inclusion and exclusion criteria can be found in the Appendix (online only). The study was approved by the institutional review board at MSKCC and conducted in accordance with the Declaration of Helsinki and Good Clinical Practice Guidelines. Written informed consent was obtained from patients before study entry. Data were analyzed through May 11, 2018.

\section{Study Procedures}

Lenvatinib was administered orally at a dose of $24 \mathrm{mg}$ per day in 28-day cycles. Dose adherence was monitored by pill diaries. Treatment was continued until disease progression, death, unacceptable toxicity, withdrawal of consent, or the treating physician's discretion that stopping therapy was to the patient's benefit. Palliative radiation was allowed if the RECIST target lesions were not included in the radiation field (received by one patient). For patients who developed grade 3 or intolerable grade 2 toxicities, lenvatinib dosing was interrupted and restarted at a lower dose level when the adverse event had improved to a tolerable grade 2 or better. In total, there were four dose levels for lenvatinib (24, 20, 14 , and $10 \mathrm{mg}$ ). Patients were removed from the study if they experienced grade 3 toxicity at the lowest dose level or for grade 4 toxicity (excluding laboratory abnormalities judged to be non-life threatening).

\section{MYB Immunohistochemistry}

Paraffin sections were analyzed with the MYB antibody from Abcam (EP769Y; Cambridge, United Kingdom) directed at the $\mathrm{N}$-terminus. MYB quantification was assessed by visual estimation of percent staining by a pathologist blinded to the results of the study.

\section{MYB-NFIB Fluorescence In Situ Hybridization}

Fluorescence in situ hybridization (FISH) was carried out on paraffin-embedded 5 - $\mu \mathrm{m}$ sections using custom probes developed from bacterial artificial chromosomes covering and flanking the MYB and NFIB genes. Two hundred successive nuclei were examined. Detection of a sufficient break-apart signal was interpreted as a positive score.

\section{Next-Generation Sequencing}

Twenty-nine patients were evaluated using the nextgeneration sequencing MSKCC Integrated Mutation Profiling of Actionable Cancer Targets (IMPACT) platform. This is a US Food and Drug Administration-approved assay for evaluating single-nucleotide variants, indels, structural variants, and copy number changes in 468 cancer-related genes from paraffin-embedded tissues. ${ }^{20,21}$ Genomic data were analyzed through the cBio Cancer Genomics Portal. ${ }^{22,23}$

\section{Statistical Analysis}

The primary end point was overall response rate (ORR; complete response plus partial response [PR] as documented by RECIST version 1.1). ${ }^{19}$ All patients who received any protocol treatment were considered evaluable for response. We established a null hypothesis of $5 \%$ ORR and $20 \%$ as the desired ORR. To test the hypothesis that the ORR was $20 \%$ or greater ( $v$ a null hypothesis of $5 \%$ ), we used a two-stage minimax study design with a one-sided type I error of $10 \%$ and power of $90 \%$. If one response was observed in the first 18 patients enrolled onto the first stage, then the study would progress to the second stage. At the end of the trial, the study would be considered positive if four or more responses occurred out of a total of 32 patients.

Secondary end points included progression-free survival (PFS) and safety and tolerability. The Kaplan-Meier method was used to estimate PFS. PFS was measured from the start of treatment to an event (disease progression or death, whichever occurred first). Patients who were event free at the date of last follow-up were censored at that time. Patients who discontinued therapy as a result of toxicity, withdrawal of consent, or physician discretion were censored at the time of last drug therapy. Biomarker analyses were performed in an exploratory fashion.

Given the limited number of progression events on trial, we performed RECIST version 1.1 measurements on post-trial scans for patients observed at our institution and performed an event-free survival (EFS) analysis. An event was defined as RECIST progression at any time point (including after the study), initiation of new therapy, or death (whichever came first). Patients were otherwise censored at the time of last drug therapy (if on treatment or lost to follow up) or the time of last radiographic disease assessment. 
TABLE 1. Baseline Characteristics Characteristic

No. of Patients (\%)*

\begin{tabular}{lc}
\hline Age, years & \\
\hline Median & 57 \\
\hline Range & $38-73$ \\
\hline Sex
\end{tabular}

\begin{tabular}{lc}
\hline Sex & \\
\hline Male & $12(37.5)$ \\
\hline Female & $20(62.5)$ \\
\hline ECOG performance status & \\
\hline 0 & 23 \\
\hline 1 & 9 \\
\hline
\end{tabular}

Primary site of ACC

\begin{tabular}{lc}
\hline Major salivary gland & $9(28.1)$ \\
\hline Minor salivary gland & $16(50.0)$ \\
\hline $\begin{array}{c}\text { Other (breast, bronchial, lacrimal gland, } \\
\text { external auditory canal) }\end{array}$ & $7(21.9)$
\end{tabular}

\begin{tabular}{lc}
\hline Disease distribution & \\
\hline Locoregional disease only & 1 \\
\hline Locoregional and metastatic disease & 8 \\
\hline Metastatic & 31 \\
\hline Lung/pleura & 31 \\
\hline Bone & 11 \\
\hline Skin & 2 \\
\hline Brain & 3 (treated) \\
\hline Kidney & 5 \\
\hline Liver & 7 \\
\hline Pancreas & 1 \\
\hline Spleen & 1 \\
\hline Nodes & 8 \\
\hline Cervical & 2 \\
\hline Noncervical (mediastinal, hilar, subdiaphragmatic) & 7 \\
\hline
\end{tabular}

\begin{tabular}{lc}
\hline Prior therapy & 3 \\
\hline Primary chemotherapy/RT alone & 1 \\
\hline Primary RT alone (protons) & 17 \\
\hline Adjuvant RT & 4 \\
\hline Adjuvant chemotherapy/RT & 1 (breast) \\
\hline Adjuvant chemotherapy followed by RT & 15 \\
\hline Palliative RT & 4 \\
\hline Palliative chemotherapy/RT & 6 \\
\hline Patients with reirradiation to any site & 7 \\
\hline Palliative chemotherapy & 2 \\
\hline Immunotherapy & 9 (6 TKI)
\end{tabular}

Abbreviations: ACC, adenoid cystic carcinoma; ECOG, Eastern Cooperative Oncology Group; PI3K, phosphoinositide 3-kinase; RT, radiation therapy; TKI, tyrosine kinase inhibitor.

*Values are numbers and percentages, unless otherwise indicated.

\section{RESULTS}

\section{Patient and Disease Characteristics}

Between June 2016 and May 2017, 33 patients were enrolled. Thirty-two patients received at least one dose of lenvatinib and were evaluable for the primary end point. One enrolled patient was removed and replaced before receiving lenvatinib and was not evaluable for the primary end point. Baseline characteristics for evaluable patients are listed in Table 1. Median age was 57 years (range, 38 to 73 years), and 12 patients (37.5\%) were men. Thirty-one patients (96.9\%) had distant metastatic disease, all of whom had lung or pleural metastases. Seven patients (22\%) also had liver metastases, and 11 patients $(34 \%)$ had bone metastases. In addition, eight patients (25\%) had both locoregional and distant metastatic disease, including two patients (6.3\%) with base of skull metastasis. Although a majority of patients (25 of 32 patients; 78\%) had ACC of either the major or minor salivary glands, seven patients had ACC from nonsalivary sites (Appendix Table A1, online only). Six patients received at least one prior VEGFR TKI. The prior TKI exposure included regorafenib alone $(n=3)$; regorafenib then axitinib ( $n=1)$; dovitinib then regorafenib $(n=1)$; and erlotinib, lapatinib, dovitinib, and then regorafenib $(n=1)$. All patients had evidence of disease progression before study participation.

\section{Efficacy}

There were four confirmed PRs in the first stage, allowing continued enrollment onto the second stage. Two patients (6.3\%) were removed before the first scan on therapy for toxicity (one for drug-related intracranial hemorrhage and another for suspected hypersensitivity reaction after the initial dose of lenvatinib). Five patients (15.6\%) achieved a confirmed PR, meeting the targeted primary end point for response. Twenty-four patients (75\%) had stable disease (SD) and one patient (3.1\%) had progression of disease as the best overall response (Table 2). The majority of patients (21 of 32 patients; 66\%) experienced tumor regression; eight (25\%) of 32 patients had $20 \%$ or greater reduction in tumor size, listed in Figure 1 . The median time on study was 5.2 months. The median PFS was 17.5 months (95\% Cl, 7.2 months to not reached), and eight progression events were observed; the remaining patients were removed for toxicity ( $n=5)$, as a result of withdrawal of consent $(n=9)$, and at the treating physician's discretion ( $n=6$ ). As of May 11, 2018, four patients remain on study treatment (duration of therapy ranges from 13.7 to 22.6 months). Of the six patients who had prior TKI exposure, one achieved a confirmed PR, three experienced minor regression, one had no change, and one had minor tumor growth.

In a post hoc EFS analysis, there were 21 events and 11 censored patients. The EFS time was 8.2 months $(95 \% \mathrm{Cl}$, 7.1 to 17.4 months; Appendix Fig A1, online only). 
TABLE 2. Lenvatinib Efficacy Measures and Reasons for Treatment Discontinuation

Efficacy Measure

No. of Patients (\%)*

\begin{tabular}{lc}
\hline Best overall response & 0 \\
\hline Complete response & $5(15.6)$ \\
\hline Partial response & $24(75)$ \\
\hline Stable disease & $1(3.1)$ \\
\hline Progression of disease & 2 (6.3) \\
\hline Off study before imaging & \\
\hline $\begin{array}{l}\text { Median progression-free survival, months } \\
(95 \% \text { Cl) }\end{array}$ & 4 (12.2 to not reached) \\
\hline Reason for discontinuation & $5(15.6)$ \\
\hline Continue on study & $6(18.8)$ \\
\hline Toxicity & $9(28.1)$ \\
\hline Physician discretion & $8(25)$ \\
\hline Withdrawal of consent
\end{tabular}

*Values are numbers and percentages, unless otherwise indicated.

\section{Toxicity, Dose Reductions, and Reasons for Discontinuation}

The most common grade 3 toxicity was hypertension in nine patients (28.1\%). Three patients had grade 3 oral pain (9.4\%). All three of these patients received adjuvant radiation as part of their primary therapy and had locally recurrent disease (oral cavity, nasopharynx, and maxillary sinus), and two of these patients had reradiation to these areas. No grade 5 toxicities were recorded. Related grade 3 and 4 toxicities are listed in Table 3.

A total of 23 patients required at least one dose reduction. Six patients underwent one dose reduction to $20 \mathrm{mg}$, nine patients underwent two dose reductions to $14 \mathrm{mg}$, and eight patients underwent three dose reductions to $10 \mathrm{mg}$. The toxicities that led to study removal included grade 4 myocardial infarction $(n=1)$, grade 4 posterior reversible encephalopathy syndrome $(n=1)$, grade 3 proteinuria $(n=$ 1 ), grade 3 orocutaneous fistula ( $n=1$; at the site of prior radiation), and grade 4 intracranial hemorrhage $(n=1)$. For the patient with intracranial hemorrhage, imaging before study entry was negative for parenchymal brain metastases. With conservative management, the patient recovered nearly to baseline with modest right peripheral vision loss. Of the six patients removed from the study as a result of physician discretion, all might have continued on protocol with a dose reduction, but drug was discontinued for grade 2 dysphagia $(n=1)$, grade 3 supraventricular tachycardia $(n=1)$, grade 3 proteinuria $(n=1)$, hypersensitivity reaction ( $n=1)$, asymptomatic grade 3 ejection fraction decrease ( $n=1$; this patient had six prior lines of therapy, and baseline ejection fraction was unknown), and tumor growth in the range of RECIST SD $(n=1)$. Nine patients withdrew consent; eight patients cited drug-related toxicities as a reason, and one patient withdrew as a result of geographic relocation. In total, 18 patients discontinued lenvatinib for reasons linked to drug toxicity.

\section{MYB Immunohistochemistry and MYBINFIB FISH}

MYB immunohistochemistry (IHC) staining was performed on 30 archival tumor specimens (two primary tumors, one local recurrence, and 27 distant metastases). Staining ranged from $0 \%$ to $80 \%$. A scatterplot of percent MYB staining versus best percent RECIST regression achieved is depicted in Figure 2. Of these samples, seven of eight patients with a response of $20 \%$ or greater had $10 \%$ of lower MYB staining, six of whom had $1 \%$ or lower MYB IHC staining. We created the following two categorical variables: PR or SD plus progression of disease. Using Fisher's exact test, MYB of $10 \%$ or less was statistically associated with PR $(P=.026)$. However, the association of MYB of $5 \%$ or less with PR was not as strongly seen $(P=.082)$.

MYB and NFIB FISH analyses were performed on 27 pretreatment samples. Eighteen samples were positive for rearrangement at both the MYB and NFIB locus, five were positive for $M Y B$ rearrangement alone, one was positive for $N F I B$ rearrangement alone, and three were negative for both. Two of the three patients who were negative for both MYB and NFIB FISH had RECIST regressions of $-53 \%$ and $-29 \%$ and had less than $1 \%$ MYB staining by $I H C$.

\section{Genomic Sequencing}

MSKCC IMPACT (468-gene next-generation sequencing platform) was performed on 29 pretreatment samples (Fig 3). The most frequently identified mutations were NOTCH1 in seven patients (24.1\%) and TERT in six patients $(20.7 \%)$. Two of the seven patients with NOTCH1 mutations experienced a confirmed PR with lenvatinib. Two patients had $4 \mathrm{q} 12$ (which encodes for PDGFRA, KIT, and $K D R$ ) amplifications (nine- and two-fold), both of whom had $\mathrm{SD}$ as a best overall response for 31 weeks $(-5 \%$ regression) and 26 weeks ( $-3 \%$ regression), respectively. Notably, the patient with nine-fold $4 q 12$ amplification was heavily pretreated with other VEGFR TKIs (dovitinib and regorafenib). One patient with a KDR (VEGFR2) E987Q mutation of unknown significance had a $13.5 \%$ regression but came off trial after 4.6 months for proteinuria. Other notable genomic alterations included a patient with a 3.8fold amplification of RET ( $-15 \%$ regression; withdrew consent after 3.3 months of treatment) and a patient with a two-fold FGFR1 amplification ( $-5 \%$ regression; came off study after 21 weeks as a result of development of an orocutaneous fistula).

\section{DISCUSSION}

The singular challenge of managing R/M ACC is the lack of effective systemic therapies. With the exception of activating NOTCH1 mutations (13\% to $33 \%$ of patients), ${ }^{11,24,25}$ the development of new treatments has been limited by the lack of common targetable genetic alterations. 


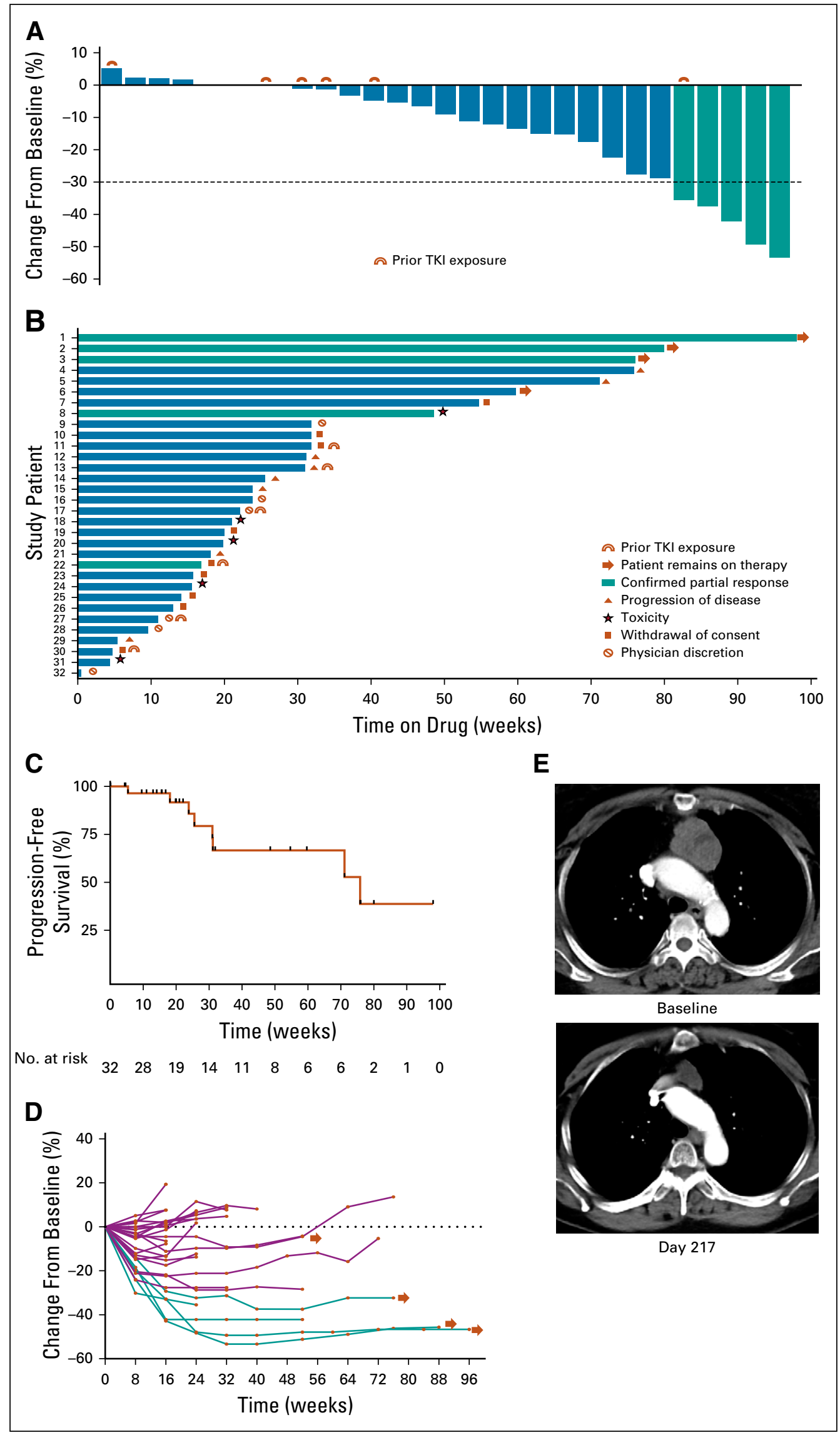

FIG 1. (A) Waterfall plot of maximum percent change in tumor size from baseline as measured by Response Evaluation Criteria in Solid Tumor (RECIST). (B) Swimmer's plot of time from start of therapy to time of last therapy, scan showing objective progression, or last known follow-up. (C) Kaplan-Meier estimate of progressionfree survival as of May 11, 2018, defined as time from first dose until objective disease progression or death from any cause; vertical lines indicate censored events. (D) Longitudinal change in RECIST percentage from baseline. (E) Pre- and posttreatment images in a patient with a partial response. Green indicates patients with confirmed partial response. Blue indicates patients with stable disease or progression of disease as best response. TKI, tyrosine kinase receptor. 
TABLE 3. Grade 3 or 4 Adverse Events Attributable to Lenvatinib

No. of Patients (\%) With Grade 3 or 4 Toxicity Related Toxicities

\begin{tabular}{ll}
\hline Hypertension & $9(28.1)$ \\
\hline Oral pain & $3(9.4)$ \\
\hline Acute coronary syndrome & $1(3.1)$ \\
\hline Anorexia & $1(3.1)$ \\
\hline Dehydration & $1(3.1)$ \\
\hline Fatigue & $1(3.1)$ \\
\hline Intracranial hemorrhage & $1(3.1)$ \\
\hline Left ventricular systolic dysfunction & $1(3.1)$ \\
\hline Myalgia & $1(3.1)$ \\
\hline Palmar-plantar erythrodysesthesia & $1(3.1)$ \\
\hline Thrombocytopenia & $1(3.1)$ \\
\hline Proteinuria & $1(3.1)$ \\
\hline Posterior reversible & $1(3.1)$ \\
\hline leukoencephalopathy syndrome & $1(3.1)$ \\
\hline Oral cutaneous fistula & $1(3.1)$
\end{tabular}

The results reported here with the multitargeted TKI lenvatinib represent promising activity in R/M ACC, regardless of genomic status. The study exceeded the prespecified primary end point, with a 15.6\% ORR (five of 32 confirmed PRs) and a median PFS of 17.5 months $(95 \% \mathrm{Cl}$, 7.2 months to not reached). These represent the best outcomes observed to date among all the VEGFR-targeting TKI studies reported in ACC (data from sunitinib, ${ }^{2}$ sorafenib, ${ }^{3,4}$ axitinib, ${ }^{5}$ dasatinib, ${ }^{6}$ regorafenib, ${ }^{7}$ and dovitinib $^{8,9}$ studies are listed in Appendix Table A2). Although cross-trial comparisons of single-institution phase II trials must be approached with caution, the phase II study of axitinib, which enrolled patients with R/M ACC at the same single center (MSKCC) using the same eligibility

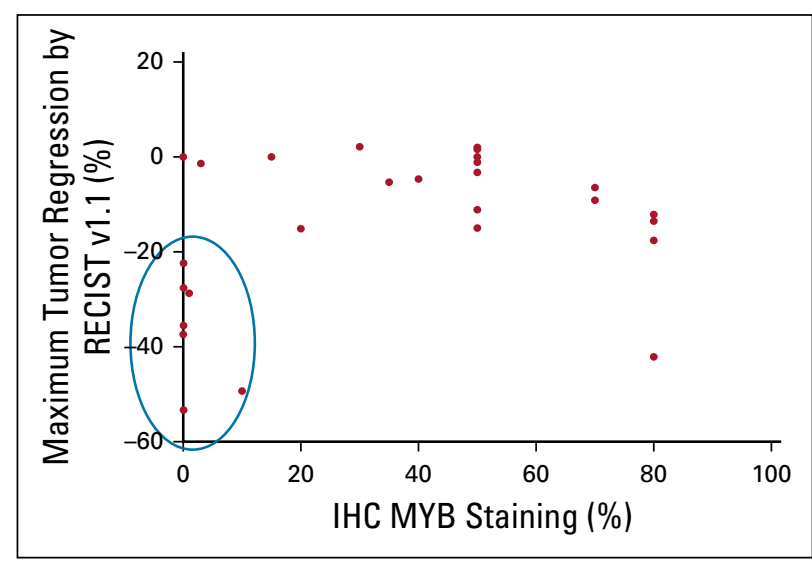

FIG 2. Relationship between MYB expression and maximum tumor regression achieved. IHC, immunohistochemistry; RECIST, Response Evaluation Criteria in Solid Tumor. criteria reported less activity than what was achieved with lenvatinib ${ }^{5}$ (axitinib $v$ lenvatinib: ORR, 9.1\% v 15.6\%; PFS, $4.8 \vee 17.1$ months, respectively). Indeed, only two prior R/M ACC TKI trials were deemed positive, both of which were sorafenib studies, and neither required disease progression as an entry criterion. ${ }^{3,4}$ Lenvatinib responses were quite durable; three of five patients have maintained PRs while continuing active treatment (time on study range, 17.5 to 22.6 months). Clinical benefit (PR and SD achieved for 6 months or greater) was achieved in $13(40.6 \%)$ of 32 patients for 6 or more months. Of six patients with prior TKI exposure, one had a confirmed PR (previously minor regression on regorafenib, which was discontinued for skin toxicity). One limitation of all single-arm phase II ACC trials is the difficulty of interpreting the true clinical significance of the PFS that is measured given the heterogeneity of disease aggressiveness among enrolled patients, differing enrollment criteria between studies, and variability in PFS methodology (Appendix Table A2). In addition, an intent-totreat PFS analysis was not possible for some patients on this study because they were not monitored for progression after treatment discontinuation. The post hoc EFS (8.2 months) analysis was performed to provide a more conservative, pragmatic estimate of the degree to which lenvatinib stalled clinically significant disease progression (events were defined as progression at any time point, initiation of any subsequent therapy, or death).

The benefits of therapy certainly must be evaluated in the context of treatment risks. Among treated patients, 62.5\% of patients had at least one grade 3 or 4 toxicity, comparable to the $75.9 \%$ rate reported in the phase III Study of (E7080) Lenvatinib in Differentiated Cancer of the Thyroid in radioiodine-refractory thyroid cancer. ${ }^{26}$ The most common grade 3 or greater toxicity observed was hypertension $(28.1 \%)$, and there were three rare but serious grade 4 events (myocardial infarction, posterior reversible encephalopathy syndrome, and intracranial hemorrhage). Eight patients (25\%) withdrew consent from the study before progression for drug-related issues. Discontinuation for drug-related reasons may be a reflection of the heavily pretreated population enrolled; of the 32 patients enrolled onto the trial, seven patients (22\%) had received chemoradiation, 16 patients (50\%) had received systemic therapy for R/M ACC (including two patients with prior immunotherapy), seven patients (22\%) had received multiple lines of therapy (including one patient with two lines of immunotherapy), and six patients (19\%) had prior multitargeted TKI exposure. Proactive management of toxicities is essential to maximize the potential clinical benefit achieved with lenvatinib.

Developing biomarkers that effectively identify patients who might benefit from lenvatinib represents an important approach to optimizing use of this therapy. So far, the search for predictive biomarkers for TKI use in ACC has yielded disappointing results. In our previous phase II trial 


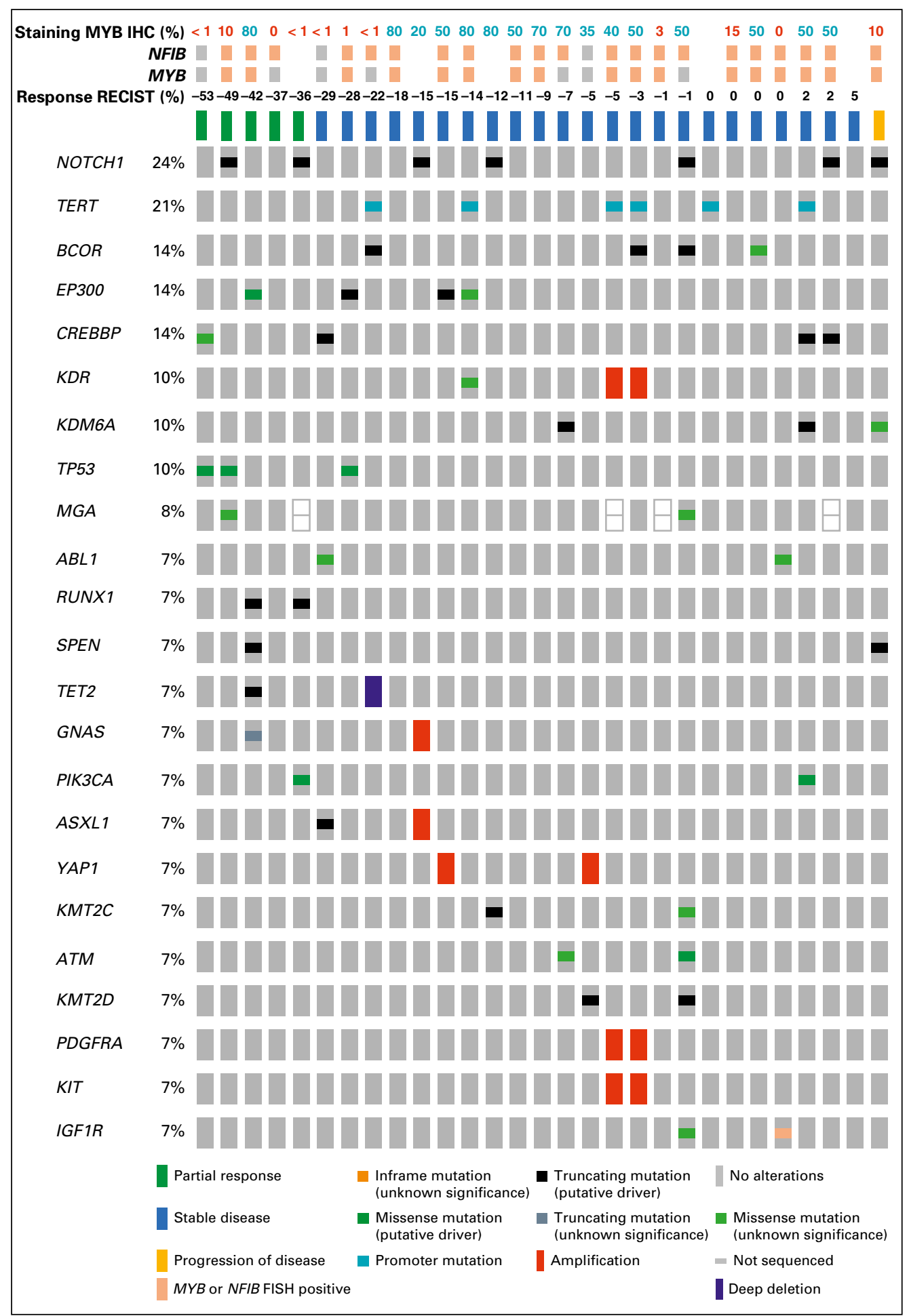

FIG 3. Integrated genomic and clinical response data. IHC, immunohistochemistry; RECIST, Response Evaluation Criteria in Solid Tumor. FISH, fluorescence in situ hybridization. of axitinib in ACC, three patients had $4 q 12$ amplifications, of whom two achieved SD for more than 6 months, including one who had a significant tumor reduction and the longest PFS on trial (21.8 months). ${ }^{5}$ In our current study, two patients with $4 \mathrm{q} 12$ amplifications had modest tumor reductions (with $-5 \%$ in the patient with nine-fold amplification and $-3 \%$ in the patient with two-fold amplification). Of note, the patient with nine-fold amplification had experienced progression through two prior lines of TKIs (dovitinib and regorafenib), which may have blunted lenvatinib efficacy. One patient had a nonhotspot mutation in the kinase insertion domain of KDR (VEGFR2) and experienced a $13.5 \%$ tumor regression (removed after 20 weeks for proteinuria). A patient with metastatic colon 
cancer with an alteration in the same VEGFR2 domain experienced an exceptional response to the VEGFRtargeted TKI regorafenib after progression on fluorouracil and bevacizumab. ${ }^{27}$ As for alterations in other genetic targets relevant to lenvatinib, one patient with a 3.8-fold RET amplification and one patient with a two-fold FGFR1 amplification experienced $15 \%$ and $5 \%$ tumor regressions, respectively. Although these genetic alterations may have contributed to therapeutic susceptibility, each represents a rare event in ACC, and they are not pragmatic markers to guide therapy for most patients.

MYB overexpression (with or without the genetic rearrangement) was hypothesized to be a potentially useful biomarker for response given that MYB is an oncogenic transcription factor that potentially mediates upregulation of several lenvatinib gene targets. ${ }^{10}$ However, we noted that patients with the greatest response by RECIST had low or no MYB expression by IHC. In addition, among three patients lacking an MYB rearrangement by $\mathrm{FISH}$, two had $1 \%$ or lower MYB protein staining by $\mathrm{IHC}$, and both achieved

\section{AFFILIATIONS}

${ }^{1}$ Memorial Sloan Kettering Cancer Center, New York, NY

${ }^{2}$ Weill Cornell Medical College, New York, NY

${ }^{3}$ Flatiron Health, New York, NY

\section{CORRESPONDING AUTHOR}

Alan L. Ho, MD, PhD, Department of Medicine, Memorial Sloan Kettering Cancer Center, 885 Second Ave, 10th Floor, New York, NY 10017; e-mail: hoa@mskcc.org.

\section{PRIOR PRESENTATION}

Presented in part at 54th Annual Meeting of the American Society of Clinical Oncology, Chicago, IL, June 1-5, 2018.

\section{SUPPORT}

Supported by Eisai. Also supported in part through the National Institutes of Health $(\mathrm{NIH}) /$ National Cancer Institute $(\mathrm{NCl})$ Cancer Center Support Grant No. P30 CA008748 and NIH/NCl Grant No. R01 CA166978. At Memorial Sloan Kettering Cancer Center, support was also provided by the Geoffrey Beene Cancer Research Center, Cycle for Survival, and the Overman Fund. The Memorial Sloan Kettering Integrated Genomics Operation Core used here for the molecular analyses was funded by $\mathrm{NCl}$ Cancer Center Support Grant No. P30 CA08748 and Cycle for Survival. excellent degrees of tumor regressions $(-53 \%$ and $-29 \%$ ). Although investigating these associations certainly can be confounded by sampling error, technical issues related to the type of tissues tested (surgical specimens, core biopsies, and fine-needle aspirations), and the small sample size, at minimum, these data suggest the hypothesis that lenvatinib activity in ACC is not reliant on MYB overexpression within the tumor.

In summary, lenvatinib elicited promising efficacy in R/M ACC. A limitation of this study is the single-arm design, because deciphering the benefits of treatment is difficult without a placebo control, and future randomized trials are warranted. The ongoing challenge is to optimize patient selection for therapy and manage drug toxicities to preserve quality of life and safety while maximizing therapeutic benefit. Genomic profiling has limited utility for predicting lenvatinib response. Future studies must continue to identify novel biomarkers that will help optimize how potentially effective therapeutics are used for these patients.
AUTHORS' DISCLOSURES OF POTENTIAL CONFLICTS OF INTEREST AND DATA AVAILABILITY STATEMENT

Disclosures provided by the authors and data availability statement (if applicable) are available with this article at DOI https://doi.org/10.1200/ JCO.18.01859

\section{AUTHOR CONTRIBUTIONS}

Conception and design: Lara Dunn, David G. Pfister, Alan L. Ho

Provision of study materials or patients: Eric J. Sherman, Lara Dunn, David G. Pfister

Collection and assembly of data: Vatche Tchekmedyian, Eric J. Sherman, Lara Dunn, Crystal Tran, Shrujal Baxi, Cristina R. Antonescu, Sofia S. Haque, David G. Pfister, Alan L. Ho

Data analysis and interpretation: Vatche Tchekmedyian, Eric J. Sherman, Crystal Tran, Shrujal Baxi, Nora Katabi, Cristina R. Antonescu, Irina

Ostrovnaya, Sofia S. Haque, David G. Pfister, Alan L. Ho

Manuscript writing: All authors

Final approval of manuscript: All authors

Accountable for all aspects of the work: All authors

\section{ACKNOWLEDGMENT}

We gratefully acknowledge the members of the Molecular Diagnostics Service in the Department of Pathology and the Marie-Josée and Henry R. Kravis Center for Molecular Oncology. We thank the research team at Memorial Sloan Kettering Cancer Center including, research nurses Lisa Cox and Colleen Hannon.

\section{REFERENCES}

1. Spiro RH: Distant metastasis in adenoid cystic carcinoma of salivary origin. Am J Surg 174:495-498, 1997

2. Chau NG, Hotte SJ, Chen EX, et al: A phase II study of sunitinib in recurrent and/or metastatic adenoid cystic carcinoma (ACC) of the salivary glands: Current progress and challenges in evaluating molecularly targeted agents in ACC. Ann Oncol 23:1562-1570, 2012

3. Thomson DJ, Silva P, Denton K, et al: Phase II trial of sorafenib in advanced salivary adenoid cystic carcinoma of the head and neck. Head Neck 37:182-187, 2015

4. Locati LD, Perrone F, Cortelazzi B, et al: A phase II study of sorafenib in recurrent and/or metastatic salivary gland carcinomas: Translational analyses and clinical impact. Eur J Cancer 69:158-165, 2016

5. Ho AL, Dunn L, Sherman EJ, et al: A phase II study of axitinib (AG-013736) in patients with incurable adenoid cystic carcinoma. Ann Oncol 27:1902-1908, 2016 
6. Wong SJ, Karrison T, Hayes DN, et al: Phase II trial of dasatinib for recurrent or metastatic c-KIT expressing adenoid cystic carcinoma and for nonadenoid cystic malignant salivary tumors. Ann Oncol 27:318-323, 2016

7. Ho AL, Sherman EJ, Baxi SS, et al: Phase II study of regorafenib in progressive, recurrent/metastatic adenoid cystic carcinoma. J Clin Oncol 34, 2016 (suppl 15; abstr 6096)

8. Dillon PM, Petroni GR, Horton BJ, et al: A phase II study of dovitinib in patients with recurrent or metastatic adenoid cystic carcinoma. Clin Cancer Res 23: 4138-4145, 2017

9. Keam B, Kim S-B, Shin SH, et al: Phase 2 study of dovitinib in patients with metastatic or unresectable adenoid cystic carcinoma. Cancer 121:2612-2617, 2015

10. Persson M, Andrén Y, Mark J, et al: Recurrent fusion of MYB and NFIB transcription factor genes in carcinomas of the breast and head and neck. Proc Natl Acad Sci USA 106:18740-18744, 2009

11. Ho AS, Kannan K, Roy DM, et al: The mutational landscape of adenoid cystic carcinoma. Nat Genet 45:791-798, 2013

12. Chen TY, Keeney MG, Chintakuntlawar AV, et al: Adenoid cystic carcinoma of the lacrimal gland is frequently characterized by MYB rearrangement. Eye (Lond) 31:720-725, 2017

13. Mitani Y, Li J, Rao PH, et al: Comprehensive analysis of the MYB-NFIB gene fusion in salivary adenoid cystic carcinoma: Incidence, variability, and clinicopathologic significance. Clin Cancer Res 16:4722-4731, 2010

14. Matsui J, Funahashi Y, Uenaka T, et al: Multi-kinase inhibitor E7080 suppresses lymph node and lung metastases of human mammary breast tumor MDA-MB231 via inhibition of vascular endothelial growth factor-receptor (VEGF-R) 2 and VEGF-R3 kinase. Clin Cancer Res 14:5459-5465, 2008

15. Matsui J, Yamamoto $\mathrm{Y}$, Funahashi $\mathrm{Y}$, et al: E7080, a novel inhibitor that targets multiple kinases, has potent antitumor activities against stem cell factor producing human small cell lung cancer H146, based on angiogenesis inhibition. Int J Cancer 122:664-671, 2008

16. Tohyama O, Matsui J, Kodama K, et al: Antitumor activity of lenvatinib (e7080): An angiogenesis inhibitor that targets multiple receptor tyrosine kinases in preclinical human thyroid cancer models. J Thyroid Res 2014:638747, 2014

17. Yamamoto Y, Matsui J, Matsushima T, et al: Lenvatinib, an angiogenesis inhibitor targeting VEGFR/FGFR, shows broad antitumor activity in human tumor xenograft models associated with microvessel density and pericyte coverage. Vasc Cell 6:18, 2014

18. Okamoto K, Kodama K, Takase K, et al: Antitumor activities of the targeted multi-tyrosine kinase inhibitor lenvatinib (E7080) against RET gene fusion-driven tumor models. Cancer Lett 340:97-103, 2013

19. Eisenhauer EA, Therasse P, Bogaerts J, et al: New response evaluation criteria in solid tumours: Revised RECIST guideline (version 1.1). Eur J Cancer 45: 228-247, 2009

20. Schrader KA, Cheng DT, Joseph V, et al: Germline variants in targeted tumor sequencing using matched normal DNA. JAMA Oncol 2:104-111, 2016

21. Cheng DT, Mitchell TN, Zehir A, et al: Memorial Sloan Kettering-Integrated Mutation Profiling of Actionable Cancer Targets (MSK-IMPACT): A hybridization capture-based next-generation sequencing clinical assay for solid tumor molecular oncology. J Mol Diagn 17:251-264, 2015

22. Cerami E, Gao J, Dogrusoz U, et al: The cBio Cancer Genomics Portal: An open platform for exploring multidimensional cancer genomics data. Cancer Discov 2: 401-404, 2012

23. Gao J, Aksoy BA, Dogrusoz U, et al: Integrative analysis of complex cancer genomics and clinical profiles using the cBioPortal. Sci Signal 6:pl1, 2013

24. Morris LGT, Chandramohan R, West L, et al: The molecular landscape of recurrent and metastatic head and neck cancers: Insights from a precision oncology sequencing platform. JAMA Oncol 46:114-117, 2016

25. Ferrarotto R, Mitani $Y$, Diao L, et al: Activating NOTCH1 mutations define a distinct subgroup of patients with adenoid cystic carcinoma who have poor prognosis, propensity to bone and liver metastasis, and potential responsiveness to Notch1 inhibitors. J Clin Oncol 35:352-360, 2017

26. Schlumberger M, Tahara M, Wirth LJ, et al: Lenvatinib versus placebo in radioiodine-refractory thyroid cancer. N Engl J Med 372:621-630, 2015

27. Loaiza-Bonilla A, Jensen CE, Shroff S, et al: KDR mutation as a novel predictive biomarker of exceptional response to regorafenib in metastatic colorectal cancer. Cureus 8:e478, 2016 
AUTHORS' DISCLOSURES OF POTENTIAL CONFLICTS OF INTEREST

Phase II Study of Lenvatinib in Patients with Progressive, Recurrent or Metastatic Adenoid Cystic Carcinoma

The following represents disclosure information provided by authors of this manuscript. All relationships are considered compensated. Relationships are self-held unless noted. I = Immediate Family Member, Inst = My Institution. Relationships may not relate to the subject matter of this manuscript. For more information about ASCO's conflict of interest policy, please refer to www.asco.org/rwc or ascopubs.org/jco/site/ifc.

Vatche Tchekmedyian

Stock and Other Ownership Interests: Infinity Pharmaceuticals, Halozyme, Rigel, Global Blood Therapeutics, Portola Pharmaceuticals, Biomarin

Eric J. Sherman

Consulting or Advisory Role: Eisai, Bristol-Myers Squibb, Novartis, Loxo, Cota Healthcare, Goldilocks

Research Funding: Eisai, Genentech, Plexxikon

Lara Dunn

Consulting or Advisory Role: Regeneron

Research Funding: Pfizer, Regeneron, Eisai

Shrujal Baxi

Employment: Flatiron Health

Stock and Other Ownership Interests: Flatiron Health

Sofia S. Haque

Honoraria: Advance Medical Inc

Consulting or Advisory Role: Advance Medical
David G. Pfister

Consulting or Advisory Role: Boehringer Ingelheim, Celgene, Bristol-Myers Squibb

Research Funding: Boehringer Ingelheim, AstraZeneca, Exelixis, Novartis, Medlmmune, Merck, Genentech (Inst), Eli Lilly (Inst), Bayer (Inst), Eisai (Inst), Regeneron

Alan L. Ho

Consulting or Advisory Role: Bristol-Myers Squibb, Eisai, Genzyme, Merck, Novartis, Sun Pharma, Regeneron, TRM Oncology, Ayala Pharmaceuticals, AstraZeneca, Sanofi

Speakers' Bureau: Medscape, Omniprex America, Novartis

Research Funding: Eli Lilly, Genentech, AstraZeneca, Bayer, Kura, Kolltan Pharmaceuticals, Eisai, Bristol-Myers Squibb, Astellas Pharma, Novartis, Merck, Pfizer, Ayala Pharmaceuticals, Allos Therapeutics, Daiichi Sankyo

Travel, Accommodations, Expenses: Janssen Oncology, Merck, Kura, Ignyta, Ayala Pharmaceuticals

No other potential conflicts of interest were reported. 


\section{Patient Inclusion Criteria}

- Patients must have pathologically or cytologically confirmed adenoid cystic carcinoma. Cancers arising from nonsalivary gland primary sites are allowed.

- Patients must have recurrent and/or metastatic disease not amenable to potentially curative surgery or radiotherapy.

- At least 2 weeks must have elapsed since the end of prior systemic treatment (4 weeks for bevacizumabcontaining regimens) or radiotherapy, with resolution of all treatment-related toxicity to National Cancer Institute Common Terminology Criteria for Adverse Events (NCI CTCAE) version 4.0 grade 1 or lower (or tolerable grade 2) or back to baseline (except for alopecia, lymphopenia, or hypothyroidism). Any number of prior therapies for recurrent or metastatic adenoid cystic carcinoma are allowed.

- Patients must have Response Evaluation Criteria in Solid Tumors (RECIST) version 1.1 measurable disease, defined as at least one lesion that can be accurately measured in at least one dimension (longest diameter to be recorded for non-nodal lesions and short axis for nodal lesions) as greater than $20 \mathrm{~mm}$ with conventional techniques or as greater than $10 \mathrm{~mm}$ with spiral computed tomography scan.

- Patients must have documentation of a new or progressive lesion on a radiologic imaging study performed within 6 months before study enrollment (progression of disease over any interval is allowed) and/or new or worsening disease-related symptoms within 6 months before study enrollment. Note that this assessment will be performed by the treating investigator. Evidence of progression by RECIST is not required.

- Patients must have archival tissue from the primary tumor or metastases available for correlative studies. Either a paraffin block or 20 unstained slides are acceptable. (If less than 20 unstained slides are available, the patient may be able to participate at the discretion of the investigator.)

- Age 18 years or older.

- Eastern Cooperative Oncology Group performance status less than 2 (Karnofsky performance status greater than $60 \%$; see Data Supplement).

- Adequate bone marrow, liver, and renal function (as suggested for lenvatinib studies).

- Total bilirubin of $1.5 \times$ the upper limit of normal (ULN) or lower.

- ALT and AST of $2.5 \times$ ULN or lower ( $5 \times$ ULN or lower for patients with liver involvement of their cancer).

- Alkaline phosphatase limit $2.5 \times$ ULN or lower $(5 \times$ ULN or lower for patients with liver involvement of their cancer).

- Serum creatinine $1.5 \times$ ULN or lower or calculated creatinine clearance greater than $60 \mathrm{~mL} / \mathrm{min}$.

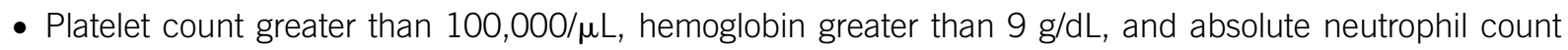
greater than $1,500 / \mu \mathrm{L}$. Blood transfusion to meet the inclusion criteria will not be allowed.

- Patients must be able to understand and be willing to sign the written informed consent form. A signed informed consent form must be appropriately obtained before the conduct of any trial-specific procedure.

- Women of childbearing potential must have a negative serum pregnancy test performed within 2 weeks before the start of study drug. Postmenopausal women (defined as no menses for at least 1 year) and surgically sterilized women are not required to undergo a pregnancy test.

- Patients (men and women) of childbearing potential must agree to use adequate contraception beginning at the signing of the consent form until at least 3 months after the last dose of study drug. The definition of adequate contraception will be based on the judgment of the principal investigator or a designated associate.

- Patient must be able to swallow and retain oral medication.

\section{Patient Exclusion Criteria}

- Concurrent anticancer therapy (chemotherapy, definitive radiation therapy, surgery, immunotherapy, biologic therapy, or tumor embolization) other than study treatment. Concurrent therapy with bisphosphonates or denosumab for bone metastases is allowed. Palliative radiation to nontarget lesions is also allowed.

- Prior use of lenvatinib.

- Uncontrolled hypertension (systolic pressure greater than $140 \mathrm{~mm} \mathrm{Hg}$ or diastolic pressure greater than $90 \mathrm{~mm} \mathrm{Hg}$ [NCl-CTCAE v4.0] on repeated measurement) despite optimal medical management. 
- Concurrent use of another investigational drug or device (ie, outside of study treatment) during or within 4 weeks of trial entry (signing of the informed consent form).

- Clinically significant proteinuria: Patients with greater than 1+ proteinuria on urinalysis will undergo 24-hour urine collection for quantitative assessment of proteinuria. Patients with urine protein of $1 \mathrm{~g}$ or greater over 24 hours will be ineligible.

- Active or clinically significant cardiac disease including the following: congestive heart failure (greater than New York Heart Association class II); active coronary artery disease that is not medically treated; cardiac arrhythmias requiring antiarrhythmic therapy other than $\beta$-blockers or digoxin; or unstable angina (anginal symptoms at rest), new-onset angina within 3 months before registration, or myocardial infarction within 6 months before registration.

- Patients with thrombotic, embolic, venous, or arterial events, such as cerebrovascular accident (including transient ischemic attacks), deep vein thrombosis, or pulmonary embolism within 6 months of study treatment start.

- Symptomatic metastatic brain or leptomeningeal tumors (asymptomatic or treated metastatic brain or leptomeningeal tumors are allowed).

- Major surgical procedure, open biopsy, or significant traumatic injury within 28 days before start of study medication.

- Evidence or history of bleeding diathesis or coagulopathy.

- Any hemorrhage or bleeding event $\mathrm{NCI}$ CTCAE grade 3 or greater within 4 weeks before start of study medication.

- Therapeutic anticoagulation with vitamin $\mathrm{K}$ antagonists (eg, warfarin) is not allowed if the medication dose and/or international normalized ratio and partial thromboplastin time are not considered stable by the treating physician. If the dose and/or international normalized ratio and partial thromboplastin time are stable, therapeutic anticoagulation with vitamin $\mathrm{K}$ antagonists is allowed with close monitoring. Anticoagulation with heparin or heparinoids is allowed.

- Known history of human immunodeficiency virus (HIV) infection or current chronic or active hepatitis B or C infection requiring treatment with antiviral therapy.

- Active infection that would impair the ability of the patient to receive study treatment.

- Presence of a nonhealing wound or nonhealing ulcer that is not tumor related.

- Renal failure requiring hemodialysis or peritoneal dialysis.

- Patients with seizure disorder requiring medication.

- Interstitial lung disease with ongoing signs and symptoms at the time of informed consent.

- History of organ allograft (including corneal transplantation).

- Any malabsorption condition.

- Women who are pregnant or breastfeeding.

- Any condition that, in the investigator's opinion, makes the patient unsuitable for trial participation. 


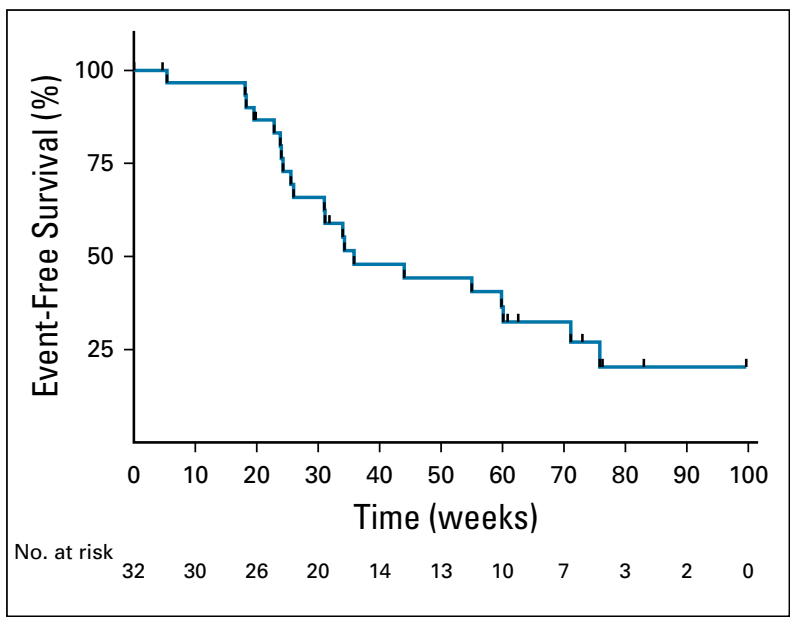

FIG A1. Kaplan-Meier estimate of event-free survival as of May 11, 2018. An event was defined as Response Evaluation Criteria in Solid Tumors (RECIST) progression at any time point (including after study), initiation of new therapy, or death (whichever came first). Patients were otherwise censored at the last day of therapy (if still on study) or the last day of therapy on study if lost to follow-up. 
TABLE A1. Primary Sites and Sites of Locoregional Tumor

Tumor Site

No. of Patients (\%)

\begin{tabular}{|c|c|}
\hline \multicolumn{2}{|l|}{ Primary site } \\
\hline \multicolumn{2}{|l|}{ Major salivary gland } \\
\hline Parotid & 6 \\
\hline Submandibular & 2 \\
\hline Sublingual & 1 \\
\hline \multicolumn{2}{|l|}{ Minor salivary gland } \\
\hline Maxillary sinus & 4 \\
\hline Palate & 3 \\
\hline Floor of mouth & 2 \\
\hline Base of tongue & 1 \\
\hline Larynx & 1 \\
\hline Nasopharynx & 3 \\
\hline Nasal cavity & 2 \\
\hline \multicolumn{2}{|l|}{ Other } \\
\hline Breast & 2 \\
\hline Bronchial & 2 \\
\hline External auditory canal & 1 \\
\hline Lacrimal gland & 1 \\
\hline Unknown & 1 \\
\hline \multicolumn{2}{|l|}{ Sites of locoregional tumor } \\
\hline Breast & 2 \\
\hline Maxillary sinus & 1 \\
\hline Cervical nodes & 1 \\
\hline Oral cavity & 1 \\
\hline Floor of mouth & 1 \\
\hline Skull base & 2 \\
\hline Auditory canal & 1 \\
\hline
\end{tabular}


TABLE A2. Phase II Trials of Multitargeted Tyrosine Kinase Inhibitors in Adenoid Cystic Carcinoma

\begin{tabular}{|c|c|c|c|c|}
\hline Study Agent & $\begin{array}{c}\text { Response Rate } \\
\text { (\% [no. of patients/total no.]) }\end{array}$ & $\begin{array}{l}\text { Median PFS or TTP } \\
\text { (months; } 95 \% \mathrm{CI} \text { ) }\end{array}$ & $\begin{array}{l}\text { Progression of } \\
\text { Disease } \\
\text { Required? }\end{array}$ & $\begin{array}{c}\text { PFS/TTP Criteria and No. of Patients } \\
\text { Censored }\end{array}$ \\
\hline Sunitinib ${ }^{2}$ & $0(0 / 14)$ & 7.2 (2.2 to 9.0) (TTP) & Yes & No patients censored in TTP analysis. \\
\hline Sorafenib ${ }^{4}$ & $11(2 / 19)$ & 8.9 (95\% Cl not reported) (PFS) & No & $\begin{array}{l}\text { PFS defined as time of initiation to } \\
\text { progression or death; patients who were } \\
\text { event free were censored (unclear of No. } \\
\text { of censored patients). No patients } \\
\text { remain on therapy. }\end{array}$ \\
\hline
\end{tabular}

\begin{tabular}{|c|c|c|c|c|}
\hline Sorafenib ${ }^{3}$ & $11(2 / 19)$ & 11.3 (8.9 to 13.7 ) (PFS) & No & $\begin{array}{l}\text { PFS defined as time of initiation to } \\
\text { progression or death; alive patients who } \\
\text { stopped drug for toxicity were censored } \\
\text { at time of study drug discontinuation. } \\
\text { Two (11\%) of } 19 \text { patients censored for } \\
\text { nonprogression events. However, for an } \\
\text { additional six (32\%) of } 19 \text { patients who } \\
\text { did not experience progression on study } \\
\text { (who came off for toxicity or intercurrent } \\
\text { illness), date of death was used as the } \\
\text { PFS event. No patients remain on } \\
\text { therapy. }\end{array}$ \\
\hline Dovitinib $^{8}$ & $6(2 / 34)$ & 8.2 (7.3 to 11) (PFS) & Yes & $\begin{array}{l}\text { Unclear methodology for calculation of PFS } \\
\text { or use of censorship. However, five } \\
(15 \%) \text { of } 34 \text { patients were censored. } \\
\text { Unclear if any remain on therapy. }\end{array}$ \\
\hline Dovitinib $^{9}$ & $3.1(1 / 32)$ & 6.0 (4.4 to 7.6) (PFS) & Yes & $\begin{array}{l}\text { PFS defined as start of therapy to } \\
\text { progression or death. No discussion of } \\
\text { censorship. However, the text states } \\
\text { there were } 17 \text { progression events, nine } \\
\text { patients who came off for toxicity, four } \\
\text { who withdrew consent, and two who } \\
\text { remained on therapy. }\end{array}$ \\
\hline Axitinib $^{5}$ & $9.1(3 / 33)$ & 5.7 (5.3 to 9.1) (PFS) & Yes & $\begin{array}{l}\text { PFS defined as start of therapy to } \\
\text { progression or death; patients censored } \\
\text { for toxicity or withdrawal for } \\
\text { nonprogression events. Seven (21\%) of } \\
33 \text { patients censored. No patients } \\
\text { remain on therapy. }\end{array}$ \\
\hline Regorafenib $^{7}$ & $0(0 / 38)$ & - & Yes & PFS not provided. \\
\hline Dasatinib $^{6}$ & $2.5(1 / 40)$ & 4.8 (1.8 to 6.9) (PFS) & Yes & $\begin{array}{l}\text { PFS defined as start of therapy to } \\
\text { progression. Six (15\%) of } 40 \text { patients } \\
\text { censored, although unclear which } \\
\text { patients were censored and at what date. } \\
\text { No patients remain on therapy. }\end{array}$ \\
\hline Lenvatinib & $15(5 / 32)$ & 17.5 (7.2 to NR) (PFS) & Yes & $\begin{array}{l}\text { PFS defined as start of therapy to } \\
\text { progression or death; patients censored } \\
\text { at time of last therapy if off therapy as } \\
\text { a result of toxicity, withdrawal of consent, } \\
\text { or physician discretion or at time of last } \\
\text { follow-up if still on study. Twenty-four } \\
\text { patients (74\%) were censored, with four } \\
\text { remaining on therapy. }\end{array}$ \\
\hline
\end{tabular}

Abbreviations: NR, not reached; PFS, progression-free survival; TTP, time to progression. 